\title{
The Ring-Shaped CMOS-Based Phototransistor With High Responsivity for the UV/Blue Spectral Range
}

\author{
Yu-Wei Chang and Yang-Tung Huang, Member, IEEE
}

\begin{abstract}
The ring-shaped phototransistor with a floating bulk enclosed by a ring-shaped photodiode is proposed to enhance the responsivity for the ultraviolet/blue spectral range. The P-channel metal-oxide-semiconductor field-effect transistor and N-channel metal-oxide-semiconductor field-effect transistor phototransistors were manufactured using a standard $0.35-\mu \mathrm{m}$ complimentary metal-oxide-semiconductor (CMOS) technology. When the phototransistors were illuminated with $400-\mathrm{nm}$ light, the measurement results for $3-\mathrm{V}$ bias demonstrated a responsivity higher than $1500 \mathrm{~A} / \mathrm{W}$, which is also superior to that of other reported photodetectors manufactured using a standard CMOS technology. Even for very small bias voltages such as $0.1 \mathrm{~V}$, the phototransistor can exhibit a responsivity of $17.9 \mathrm{~A} / \mathrm{W}$.
\end{abstract}

Index Terms-Optical receivers, optoelectronic devices, photodetectors, phototransistors.

\section{INTRODUCTION}

$\mathbf{T}$ HE development of a photodetector with high responsivity for the ultraviolet (UV)/blue spectral range has attracted much interest for many applications, such as biochemical analysis and optical storage systems [1]-[4]. For biochemical analysis, the detection of the optical properties changed by chemical reactions is a competent approach to examine various important biological molecules. Through the chemical reactions, the change of the emitted - or absorbed-light intensity is generally less than $1 \mu \mathrm{W}$. Regarding the reported methods, the luminol $/ \mathrm{H}_{2} \mathrm{O}_{2} / \mathrm{HRP}$ reaction involves the emission of 425-nm light [1], and the ABTS $/ \mathrm{H}_{2} \mathrm{O}_{2} / \mathrm{HRP}$ reaction involves the absorption of 415-nm light [2]. For optical storage systems, an optical pickup unit detects the laser light (less than $1 \mu \mathrm{W}$ ) reflected from a storage disc and converts it into electrical output signals [3]. The recording density of the disc is determined by the minimum spot size that the laser light can be focused on, and the spot size is proportional to the laser wavelength. Therefore, a higher recording density can be obtained by using the laser of a shorter wavelength [4]. Compared with CD $(780 \mathrm{~nm})$ and DVD $(650 \mathrm{~nm})$, the Blu-ray Disc $(405 \mathrm{~nm})$ has a larger storage capacity and becomes a noticeable trend in the optical storage technology.

Much work has been devoted to the realization of photodetectors using a standard complimentary metal-oxide-semiconductor (CMOS) technology or CMOS-compatible technologies

Manuscript received December 05, 2008; revised March 20, 2009. First published April 17, 2009; current version published June 17, 2009.

The authors are with the Department of Electronics Engineering and Institute of Electronics, National Chiao Tung University, Hsinchu City 300, Taiwan (e-mail: jameschang.ee93g@nctu.edu.tw).

Digital Object Identifier 10.1109/LPT.2009.2020176
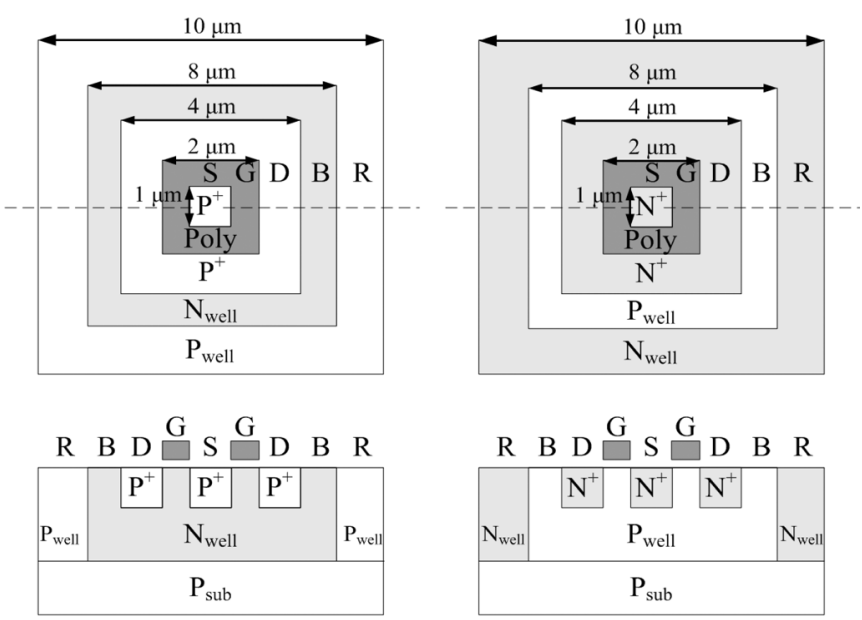

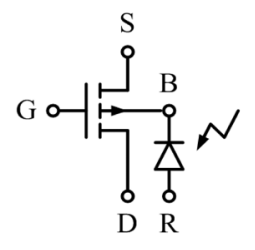

(a)

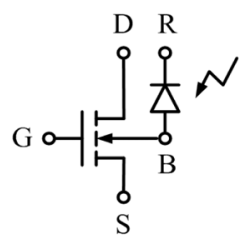

(b)
Fig. 1. Top view, cross section, and equivalent symbol of the proposed (a) PMOS phototransistor and (b) NMOS phototransistor.

in the last decade [1]-[11]. This is owing to its low cost, high throughput, and the capability for integration with consequent circuits without any process modification. A conventional CMOS photodiode can easily be formed by utilizing an $\mathrm{N}^{+} / \mathrm{P}_{\text {well }}, \mathrm{P}^{+} / \mathrm{N}_{\text {well }}$, or $\mathrm{N}_{\text {well }} / \mathrm{P}_{\text {sub }}$ junction, but the responsivity for the UV/blue spectral range is low (about $0.2 \mathrm{~A} / \mathrm{W}$ ) [12]. Circumventing this drawback, a phototransistor can have a current amplification under moderate bias through the internal transistor action [13]. However, little work has been conducted on the phototransistor manufactured using a standard CMOS technology for the UV/blue spectral range.

In this research, the CMOS phototransistor was designed, fabricated, and measured. It exhibited an enhanced responsivity for the UV/blue spectral range.

\section{Device Structure and Operating Principle}

The device structures of the proposed P-channel metal-oxide-semiconductor field-effect transistor (PMOS) and $\mathrm{N}$-channel metal-oxide-semiconductor field-effect transistor (NMOS) phototransistor based on CMOS technology are shown in Fig. 1(a) and (b), respectively. For the PMOS phototransistor, the $\mathrm{P}^{+}$source is placed in the center with the 
ring-shaped poly-silicon gate and $\mathrm{P}^{+}$drain, while the $\mathrm{N}_{\text {well }}$ bulk $(B)$ is enclosed by the $\mathrm{P}_{\text {well }}$ ring $(R)$. For the NMOS phototransistor, the $\mathrm{N}^{+}$source is placed in the center with the ring-shaped poly-silicon gate and $\mathrm{N}^{+}$drain, while the $\mathrm{P}_{\text {well }}$ bulk $(B)$ is enclosed by the $N_{\text {well }}$ ring $(R)$. In both cases, the phototransistor can be regarded as a metal-oxide-semiconductor field-effect transistor (MOSFET) with a photodiode connected across the bulk $(B)$ and the square ring $(R)$, as shown in Fig. 1.

Serving as an optical sensor, the phototransistor leaves the bulk floating and mainly uses the $\mathrm{N}_{\text {well }} / \mathrm{P}_{\text {well }}$ junction to separate photogenerated electron-hole pairs [8]. The photogenerated carriers drifting into the bulk would change the bulk potential, and thereby change the threshold voltage as well as the output drain current.

For the UV/blue incident light, most photogenerated carriers are very close to the surface (within $0.2 \mu \mathrm{m}$ ). Using the proposed ring-shaped layout style, the near-surface depletion region of the outer $\mathrm{N}_{\text {well }} / \mathrm{P}_{\text {well }}$ ring-shaped photodiode is enlarged and superior to that of a conventional MOSFET with the same area, so as to cause a larger shift amount of the threshold voltage. Hence a larger photocurrent response $I_{\mathrm{ph}}$ could be expected.

Regarding the proposed phototransistors, the bulk is left floating, while the applied fixed ring-source voltage $V_{\mathrm{RS}}$ is negative for PMOS and is positive for NMOS. The bulk-ring voltage $V_{\mathrm{BR}}$ resulting from the illumination can be described by the transposed diode equation [13]

$$
V_{\mathrm{BR}}=V_{\mathrm{th}} \ln \left(1+\frac{I_{\mathrm{BR}(\mathrm{ph})}}{I_{\mathrm{BR} 0}}\right)=V_{\mathrm{th}} \ln \left(1+\frac{k_{1} P_{\mathrm{opt}}}{I_{\mathrm{BR} 0}}\right)
$$

where $V_{\mathrm{th}}, I_{\mathrm{BR}(\mathrm{ph})}, I_{\mathrm{BR} 0}$, and $P_{\mathrm{opt}}$ are the thermal voltage, the photocurrent of the bulk-ring diode, the saturation current of the bulk-ring diode, and the incident optical power density, respectively; $k_{1}$ is functions of material parameters, transistor bias voltages, and the depletion region area of the $\mathrm{N}_{\text {well }} / \mathrm{P}_{\text {well }}$ junctions. Thus the resulted bulk-source voltage $V_{\mathrm{BS}}$ can be expressed as $V_{\mathrm{BS}}=V_{\mathrm{BR}}+V_{\mathrm{RS}}$. For illuminated PMOS, $V_{\mathrm{BS}}$ is negative since its $\mathrm{N}_{\text {well }}$ bulk accumulates excess electrons; for illuminated NMOS, $V_{\mathrm{BS}}$ is positive since its $\mathrm{P}_{\text {well }}$ bulk accumulates excess holes.

The shift amount of the threshold voltage $\Delta V_{T}$ due to the bulk charge effect is given by [13]

$$
\Delta V_{T}=\frac{\sqrt{2 \varepsilon_{s} q N_{B}}}{C_{\mathrm{ox}}}\left(\sqrt{2 \psi_{B}-V_{\mathrm{BS}}}-\sqrt{2 \psi_{B}}\right)
$$

where $\varepsilon_{s}, q, N_{B}, C_{\mathrm{ox}}$, and $\psi_{B}$ are the permittivity of silicon, the unit electric charge, the doping concentration of the bulk, the oxide capacitance per unit area, and the bulk Fermi level from the intrinsic Fermi level, respectively. When PMOS and NMOS phototransistors are illuminated, the absolute value of the threshold voltage $\left|V_{T}\right|$ will both become smaller.

The photocurrent response $I_{\mathrm{ph}}$ is the difference in the output drain current, which can be expressed as

$$
I_{\mathrm{ph}}=\left|I_{D}-I_{D 0}\right|
$$

where $I_{D}$ and $I_{D 0}$ denote the output drain current of the phototransistor with and without illumination, respectively. It implies

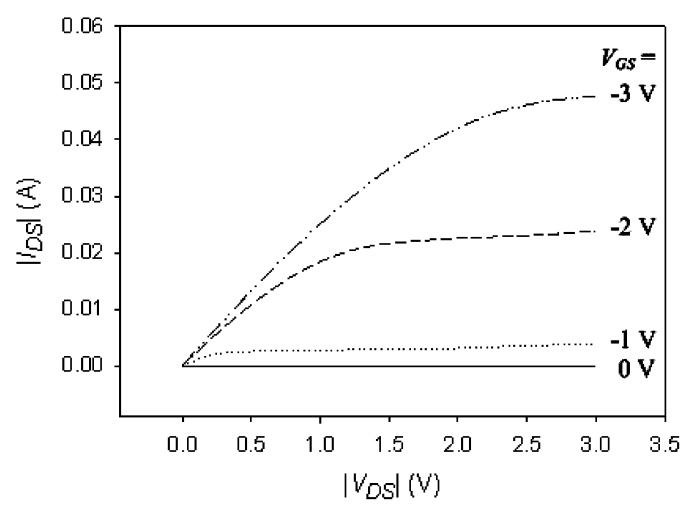

Fig. 2. Measured $I-V$ characteristics of the PMOS phototransistor without illumination.

that $I_{\mathrm{ph}}$ of the phototransistor will increase with the $\mathrm{N}_{\text {well }} / \mathrm{P}_{\text {well }}$ sensing area $A$, the channel aspect ratio $W / L$, and the bias voltages $\left|V_{\mathrm{GS}}\right|$ and $\left|V_{\mathrm{DS}}\right|$.

Overall, with advantages of the ring-shaped structure and the MOSFET amplification, the proposed CMOS phototransistor is expected to perform a better responsivity than conventional CMOS-based photodetectors.

\section{EXPERIMENTAL RESULTS}

The proposed PMOS and NMOS phototransistors were respectively duplicated and concatenated to form each $10 \times 10$ array with a total area of $100 \mu \mathrm{m} \times 100 \mu \mathrm{m}$, and they were manufactured using the Taiwan Semiconductor Manufacturing Company (TSMC) $0.35-\mu \mathrm{m}$ standard CMOS technology. To characterize these phototransistors, a UV/blue LED (Centenary 40327) with a peak of $400 \mathrm{~nm}$ was used as the light source, while its light intensity was adjusted by a power supply (Agilent E3631A). A reference photodetector and a pinhole were introduced to measure the optical power density for various bias voltages applied to the LED. A precision semiconductor parameter analyzer (HP 4156A) and a test fixture (HP 16058A) were used to characterize the CMOS photodetectors.

Fig. 2 shows the measured current-voltage $(I-V)$ characteristics of the PMOS phototransistor without illumination. The bias conditions were as follows: the source was grounded, the drain voltage swept from 0 to $-3 \mathrm{~V}$, the gate voltage stepped from 0 to $-3 \mathrm{~V}$, the bulk was floating, and the ring voltage was fixed at $-3 \mathrm{~V}$.

While the phototransistors were illuminated, the output drain current varied in response to the light intensity. For the PMOS phototransistor with $\left|V_{\mathrm{DS}}\right|=3 \mathrm{~V}$, the measured photocurrent responses are shown in Fig. 3(a). The larger the $\left|V_{\mathrm{GS}}\right|$ was, the larger the $\left|I_{D}\right|$ as well as the $I_{\mathrm{ph}}$ were. The photocurrent response divided by illumination intensity gives the DC responsivity, and the results are shown in Fig. 3(b). With $V_{\mathrm{GS}}=-3 \mathrm{~V}$, the phototransistor exhibited a responsivity higher than $1500 \mathrm{~A} / \mathrm{W}$ when the illumination intensity was less than $1 \mu \mathrm{W}$, as the condition for applications in optical storage and most biochemical detection. This responsivity is 7500 folds higher than that of a conventional silicon $\mathrm{P} / \mathrm{N}$ junction photodiode (about $0.2 \mathrm{~A} / \mathrm{W}$ ) [12]. In addition, when the illumination 


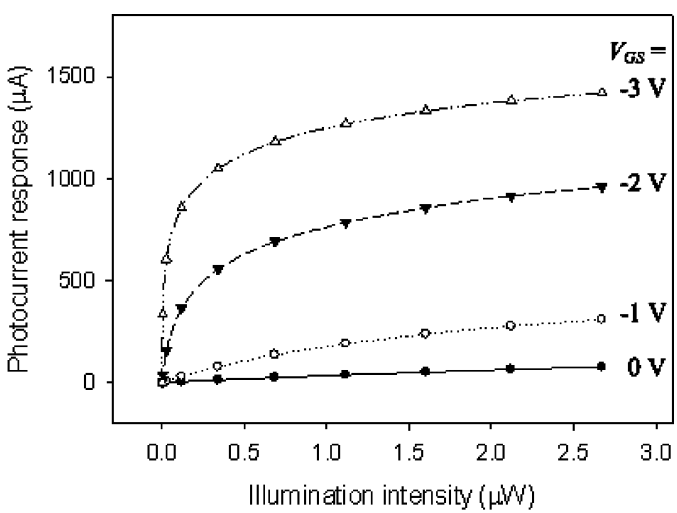

(a)

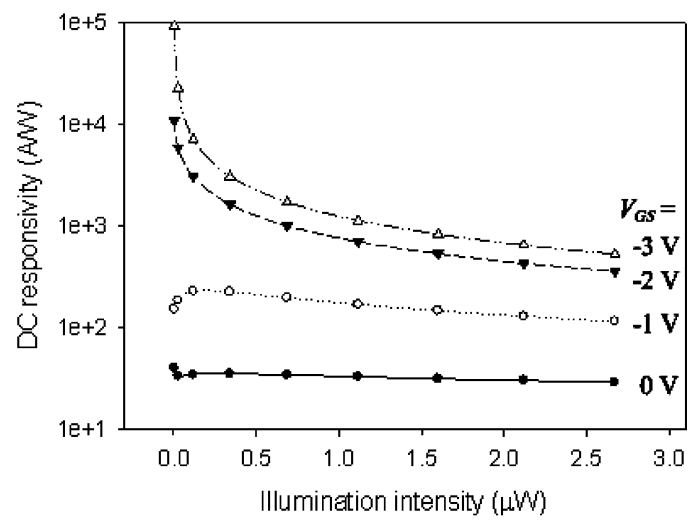

(b)

Fig. 3. Optoelectronic characteristics of the PMOS phototransistor for $V_{\mathrm{DS}}=$ $-3 \mathrm{~V}$ with various $V_{\mathrm{GS}}$ : (a) photocurrent response versus illumination intensities and (b) DC responsivity versus illumination intensities.

TABLE I

UV/BLUE RESPONSIVITY OF THE CMOS PHOTODETECTORS

\begin{tabular}{|c|c|c|c|c|}
\hline Device Type & Ref. & $\lambda(\mathrm{nm})$ & Bias (V) & $R(\mathrm{~A} / \mathrm{W})$ \\
\hline Conventional PD & [9] & 400 & 3 & 0.23 \\
\hline NPN PT & [10] & 420 & 5 & 0.25 \\
\hline Avalenche PD & [11] & 380 & 14.5 & 5.3 \\
\hline \multirow{4}{*}{ PMOS PT } & \multirow{4}{*}{$\begin{array}{l}\text { This } \\
\text { work }\end{array}$} & \multirow{4}{*}{400} & 0.1 & 17.9 \\
\hline & & & 1 & 1100 \\
\hline & & & 2 & 1700 \\
\hline & & & 3 & 1700 \\
\hline \multirow{4}{*}{ NMOS PT } & \multirow{4}{*}{$\begin{array}{l}\text { This } \\
\text { work }\end{array}$} & \multirow{4}{*}{400} & 0.1 & 2.3 \\
\hline & & & 1 & 2600 \\
\hline & & & 2 & 2900 \\
\hline & & & 3 & 1500 \\
\hline
\end{tabular}

(PD: photodiode, PT: phototransistor)

intensity was about $0.1 \mu \mathrm{W}$, a higher responsivity of $7200 \mathrm{~A} / \mathrm{W}$ can be achieved.

Even for very small bias voltages such as $V_{\mathrm{GS}}=V_{\mathrm{DS}}=$ $-0.1 \mathrm{~V}$, the phototransistor can successfully detect the change of illumination intensity and exhibited a responsivity of about $17.9 \mathrm{~A} / \mathrm{W}$. The dark current was less than $1 \mathrm{nA}$.

Table I summarizes the measured responsivities for the PMOS and NMOS phototransistors under various bias conditions $\left(V_{\mathrm{GS}}=V_{\mathrm{DS}}\right)$ with illumination of $0.7-\mu \mathrm{W} 400-\mathrm{nm}$ light. The characteristics of other reported photodetectors manufactured in a standard CMOS technology are also compared [9]-[11]. The difference between the PMOS and NMOS pho- totransistor mainly results from that the $\mathrm{N}_{\text {well }}$ bulk of PMOS is distinguished from $\mathrm{P}_{\text {sub }}$, whereas the NMOS has a larger bulk region since $\mathrm{P}_{\text {well }}$ is inherently connected with $\mathrm{P}_{\text {sub }}$. For further applications in integrated circuits or sensor arrays, the floating $\mathrm{P}_{\text {well }}$ of NMOS phototransistor can be separated from the $\mathrm{P}_{\text {sub }}$ by introducing a deep- $\mathrm{N}_{\text {well }}$. Among these CMOS photodetectors, the proposed phototransistor exhibits a higher responsivity but at the expense of bandwidth. The measured dynamic responsivities for $1 \mathrm{~Hz}, 1 \mathrm{kHz}$, and $1 \mathrm{MHz}$ were about 1700,800 , and $600 \mathrm{~A} / \mathrm{W}$, respectively. However, the bandwidth is sufficient for applications in biochemical analysis.

\section{CONCLUSION}

In this letter, the ring-shaped phototransistor with a floating bulk enclosed by a ring-shaped photodiode based on CMOS technology is proposed for applications in the UV/blue spectral range with a high responsivity. Experimental results demonstrated that the responsivity for 400-nm light was higher than $1500 \mathrm{~A} / \mathrm{W}$, which is also superior to that of other reported photodetectors manufactured in a standard CMOS process. Even for very small bias voltages such as $0.1 \mathrm{~V}$, the phototransistor can exhibit a responsivity of $17.9 \mathrm{~A} / \mathrm{W}$. The proposed device structure is therefore an attractive option in applications for the UV/blue spectral range.

\section{REFERENCES}

[1] U. Lu, B. C.-P. Hu, Y.-C. Shih, Y.-S. Yang, C.-Y. Wu, C.-J. Yuan, M.-D. Ker, T.-K. Wu, Y.-K Li, Y.-Z. Hsieh, W. Hsu, and C.-T. Lin, "CMOS chip as luminescent sensor for biochemical reactions," IEEE Sensors J., vol. 3, no. 3, pp. 310-316, Jun. 2003.

[2] Y.-W. Chang, P.-C. Yu, Y.-T. Huang, and Y.-S. Yang, "A CMOS-compatible optical biosensing system based on visible absorption spectroscopy," in IEEE Int. Conf. Electron Devices and Solid-State Circuits, 2007, vol. 2, pp. 1099-1102.

[3] J. Sturm, M. Leifhelm, H. Schatzmayr, S. Groiß, and H. Zimmermann, "Optical receiver IC for CD/DVD/blue-laser application," IEEE $J$. Solid-State Circuits, vol. 40, no. 7, pp. 1406-1413, Jul. 2005.

[4] A. Nemecek, G. Zach, R. Swoboda, K. Oberhauser, and H. Zimmermann, "Integrated BiCMOS p-i-n photodetectors with high bandwidth and high responsivity," IEEE J. Sel. Topics Quantum Electron., vol. 12, no. 6, pt. 2, pp. 1469-1475, Nov./Dec. 2006.

[5] W. Zhang, M. Chan, S. K. H. Fung, and P. K. Ko, "Performance of a CMOS compatible lateral bipolar photodetector on SOI substrate," IEEE Electron Device Lett., vol. 19, no. 11, pp. 435-437, Nov. 1998.

[6] M. A. Marwick and A. G. Andreou, "A UV photodetector with internal gain fabricated in silicon on sapphire CMOS," in IEEE Sensors Conf., 2007, pp. 535-538

[7] W. Zhang and M. Chan, "A high gain n-well/gate tied PMOSFET image sensor fabricated from a standard CMOS process," IEEE Trans. Electron Devices, vol. 48, no. 6, pp. 1097-1102, Jun. 2001.

[8] M. Schanz, W. Brockherde, R. Hauschild, B. J. Hosticka, and A. Teuner, "CMOS photosensor arrays with on-chip signal processing," in Proc. 23rd Eur. Solid-State Circuits Conf., 1997, pp. 236-239.

[9] A. Ghazi, H. Zimmermann, and P. Seegebrecht, "CMOS photodiode with enhanced responsivity for the UV/blue spectral range," IEEE Trans. Electron Devices, vol. 49, no. 7, pp. 1124-1128, Jul. 2002.

[10] A. Tibuzzi, G.-F. D. Bettaa, C. Piemontea, C. D. Natalea, A. D. Amicoa, and G. Soncinia, "High gain bipolar junction phototransistors with finger-shaped emitter for improved optical gas sensing in the blue spectral region," Sens. Actuators A, Phys., vol. 136, pp. 588-596, 2007.

[11] A. R. Pauchard, P.-A. Besse, and R. S. Popovic, "Dead space effect on the wavelength dependence of gain and noise in avalanche photodiodes," IEEE Trans. Electron Devices, vol. 47, no. 9, pp. 1685-1693, Sep. 2000.

[12] S. Donati, Photodetectors-Devices, Circuits, and Applications. Englewood Cliffs, NJ: Prentice-Hall, 2000.

[13] S. M. Sze and K. K. Ng, Physics of Semiconductor Devices, 3rd ed. Hoboken, NJ: Wiley, 2007. 